\title{
Localization of the Herpesvirus Portal
}

Michael H. C. Buch ${ }^{1}$, J. Bernard Heymann ${ }^{1}$, William W. Newcomb ${ }^{1}$, Dennis C. Winkler ${ }^{1}$ and Alasdair C. Steven ${ }^{1 *}$

${ }^{1 .}$ LSBR, NIAMS, NIH, Bethesda, MD, USA.

* Corresponding author: stevena@mail.nih.gov

Herpes simplex virus type 1 (HSV-1) contains a large double-stranded DNA genome of about 152,000 base pairs, which requires accommodation in a correspondingly massive particle. In the nucleus, viral DNA is packaged into a spherical precursor particle called the procapsid via the portal protein, pUL6, which occupies one of the procapsid's twelve vertices. Upon DNA insertion, the procapsid matures into an angular, icosahedral capsid and undergoes the subsequent steps in the viral life cycle. While the overall structure of the viral portal has been known for some time [1,2], its exact orientation and position in the context of the capsid have been controversial. Investigations have been hampered by the fact that the one portal-bearing vertex is practically visually indistinguishable from the other eleven vertices. This has led to various publications, using different technical approaches, placing the portal both inside the particle as well as in line with other capsomers and in various orientations [3-6].

Here, we employ a novel analysis method, which employs cryo electron tomography coupled with highly pure, mature capsids that we obtained through the use of a deletion mutant [7]. Three-dimensional (3D) particles were extracted from tomograms, aligned, and their twelve vertices were excised. These vertices were then compared to an average of themselves, and the one vertex with the least similarity to the average was identified as the special vertex. For an overview over the method, see Fig. 1.

Our results unambiguously place the portal inside the capsid and with its narrower 'stalk' facing outwards while maintaining close contact with the capsid floor (Fig. 2). The identified unique vertex density is also consistent with the structure of the portal itself (Fig. 2A). Furthermore, we observe a strong density located outside and offset from the portal vertex, roughly $100 \AA$ in diameter (Fig. 2B, arrow). This density may correspond to the terminase complex, which is responsible for translocating DNA into nascent capsids. The capsids we have used in this study have been shown to carry residual terminase [8].

Our developed method allowed us to overcome previous limitations in identifying the portal vertex and grants a clear view of a unique feature on an otherwise highly symmetric particle without introducing any symmetry-related bias.

\section{References:}

[1] WW Newcomb et al., J Virol 75 (2001), p. 10923.

[2] BL Trus et al., J Virol 78 (2004), p. 12668.

[3] G Cardone et al., Virology 361 (2007), p. 426.

[4] RH Rochat et al., J Virol 85 (2011), p. 1871.

[5] MF Schmid et al., PLoS Pathog 8 (2012), p. e1002961.

[6] M McElwee et al., PLoS Biol 16 (2018), p. e2006191.

[7] AR McNab et al., J Virol 72 (1998), p. 1060. 
[8] AK Sheaffer et al., J Virol 75 (2001), p. 687.

[9] Michael H. C. Buch, J. Bernard Heymann and William W. Newcomb contributed equally.
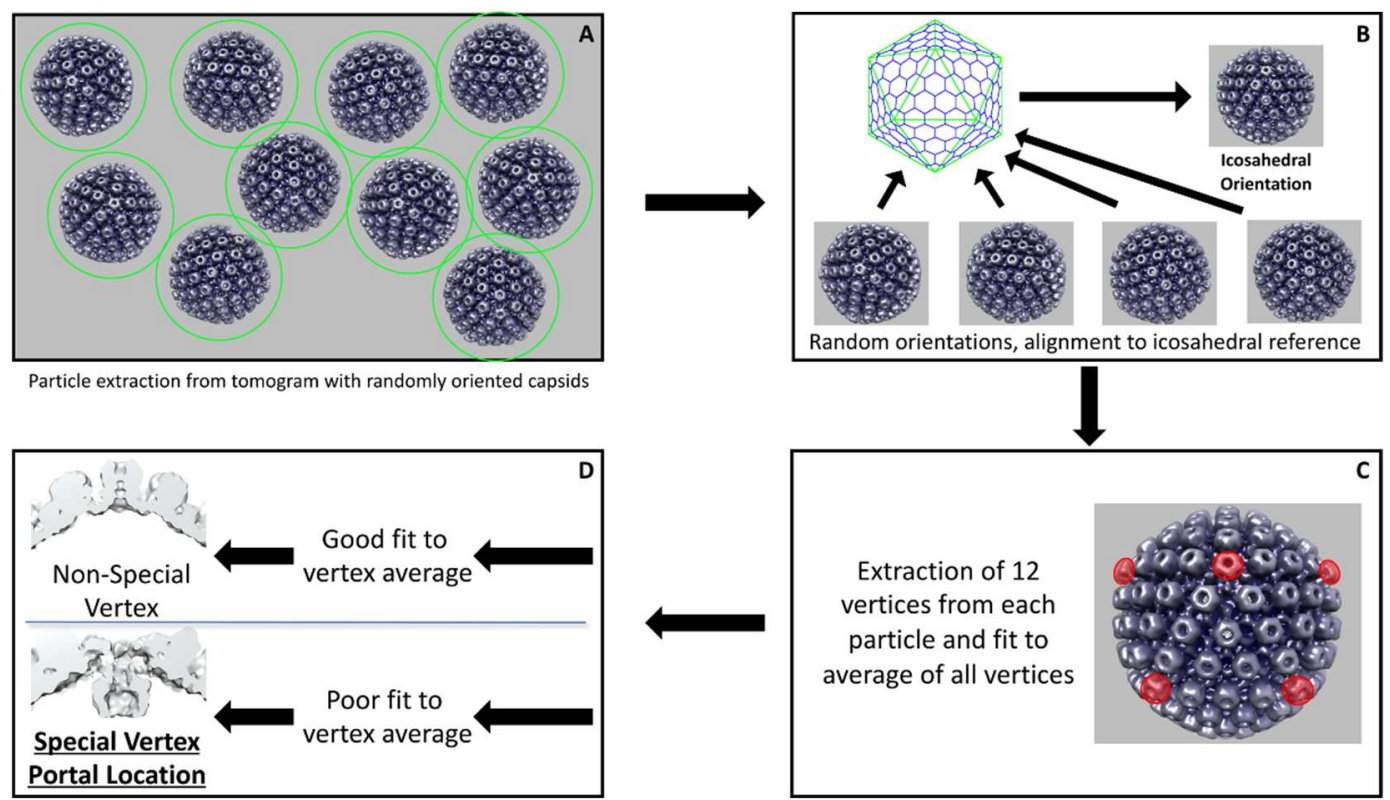

Figure 1. Workflow for unique vertex identification. Randomly oriented particles are extracted from a three-dimensional reconstruction (A) and aligned to an icosahedrally symmetric template (B). From each of these aligned particles, the twelve fivefold vertices are very narrowly excised $(C)$ and then compared to an average of the vertices. The worst fit, i.e. the vertex with the most distinct shape compared to the other eleven, is identified as the unique vertex (D).
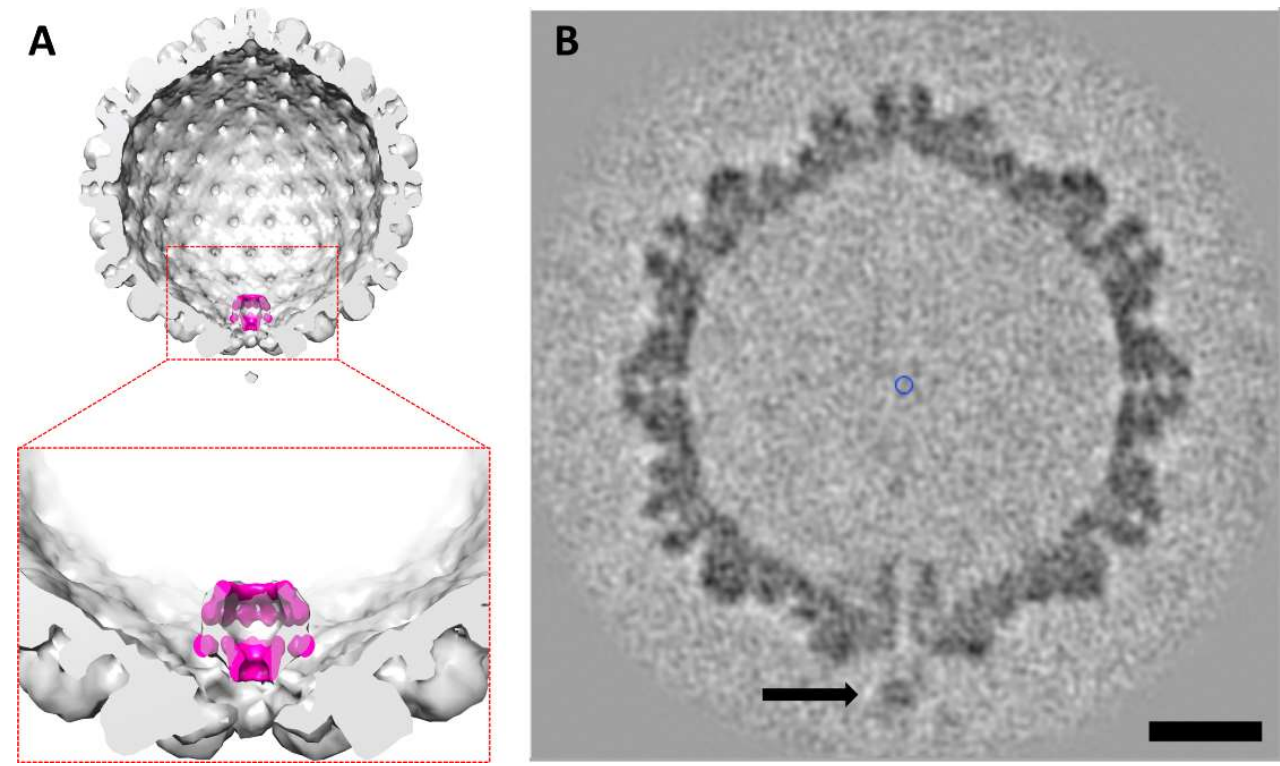

Figure 2. The portal-bearing vertex in the context of the mature capsid. The identified portal vertex was translated back into a mature capsid (A). The density clearly reaches into the inside of the particle and superposes well with a previously solved structure of the pUL6 dodecamer [2]. We also observe a striking density outside the portal vertex, which we believe to be associated with the terminase complex (B, black arrow). Scale bar, $25 \mathrm{~nm}$. 